\title{
Infrared-Phonon-Polariton Resonance of the Nonlinear Susceptibility in GaAs
}

\author{
T. Dekorsy, ${ }^{1}$ V. A. Yakovlev, ${ }^{2}$ W. Seidel,${ }^{3}$ M. Helm, ${ }^{1}$ and F. Keilmann ${ }^{4}$ \\ ${ }^{1}$ Institute of Ion Beam Physics and Materials Research, Forschungszentrum Rossendorf, P.O. Box 510119, 01314 Dresden, Germany \\ ${ }^{2}$ Institute of Spectroscopy, Russian Academy of Sciences, Troitsk, Moscow region 142190, Russia \\ ${ }^{3}$ Institute for Nuclear and Hadron Physics, Forschungszentrum Rossendorf, P.O. Box 510119, 01314 Dresden, Germany \\ ${ }^{4}$ Max-Planck-Institute für Biochemie, 82152 Martinsried, Germany
}

\begin{abstract}
Nonlinear probing of the fundamental lattice vibration of polar crystals is shown to reveal insight into higher-order cohesive lattice forces. With a free-electron laser tunable in the far infrared we experimentally investigate the dispersion of the second-order susceptibility due to the phonon resonance in GaAs. We observe a strong resonance enhancement of second harmonic light generation at half the optical phonon frequency, and in addition a minimum at a higher frequency below the phonon frequency. Measuring this frequency and comparison to a theoretical model allows the determination of competing higher-order lattice forces.
\end{abstract}

Since the invention of lasers the field of nonlinear optics evolved rapidly starting with the observation of frequency doubling of a pulsed ruby laser in crystalline quartz [1]. Since these times a multitude of materials has been investigated and developed in order to yield high second-order nonlinear susceptibilities for frequency conversion, enabling the generation of light from the mid IR to the UV covering many wavelengths where no laser source directly emits radiation. However, in the $\mathrm{THz}$ frequency range $(1-10 \mathrm{THz})$ a remarkable gap exists in the availability of tunable and intense light sources with the consequence that nonlinear optics in this frequency range remains to great extents unexplored [2]. Thus since the pioneering work of Faust and Henry on frequency mixing with a $\mathrm{HeNe}$ laser and a far-infrared (FIR) $\mathrm{H}_{2} \mathrm{O}$ laser in $\mathrm{GaP}$ [3] and microwave-mixing experiments in a variety of crystals by Boyd et al. [4] only a few experiments on the dispersion of the nonlinear susceptibility in the $\mathrm{THz}$ frequency range have been performed [5-7]. The lack of firm experimental data on a large number of technologically important crystals has been an obstacle for the development of quantitative theories. In the last decade intense free-electron lasers (FEL) tunable in the FIR have become available for the study of nonlinear optical phenomena. Recently, the first quantum cascade laser has been realized in a GaAs/AlGaAs heterostructure emitting at $4.4 \mathrm{THz}$, which may open the door for integrated nonlinear optics in this frequency range [8]. Here we report on a study of second harmonic generation (SHG) in the range of 4.0 to $6.0 \mathrm{THz}$ in thin GaAs films performed with a FEL. This experiment provides insight into the influence of higherorder terms of the lattice potential on the nonlinear susceptibility in the $\mathrm{THz}$ frequency range.

The nonlinear optical susceptibility in semiconductors in the $\mathrm{THz}$ frequency range is governed by the superposition of electronic and ionic contributions. Faust and
Henry were the first to determine the dispersion of the $\mathrm{THz}$ nonlinear susceptibility in GaP [3]. They observed a resonance enhancement at the TO phonon frequency and showed that the ionic and electronic contributions are of opposite sign, leading to a cancellation of both contributions below the phonon resonance. They showed theoretically that the pure electronic susceptibility $\chi_{E}^{(2)}$ is related to the dispersion of the susceptibility in the FIR for the case of frequency mixing via

$$
\chi^{(2)}\left(\omega_{1}-\omega, \omega, \omega_{1}\right)=\chi_{E}^{(2)}\left[1+C_{1} \frac{1}{D(\omega)}\right]
$$

with $D(\omega)=1-\left(\omega / \omega_{\mathrm{TO}}\right)^{2}-i \omega \gamma / \omega_{\mathrm{TO}}^{2}, \omega_{\mathrm{TO}}$ the TO phonon frequency, and $\gamma$ the damping of the TO phonon. $\chi_{E}^{(2)}$ is the pure electronic part of the nonlinear susceptibility and can be determined experimentally from frequency doubling above the lattice resonance, in the transparent range of the crystal [9-11]. The FaustHenry coefficient $C_{1}$, which is negative for most compound semiconductors, reflects the different signs of the electronic and ionic contributions to the nonlinear susceptibility [12]. This coefficient is further important since it defines the ratio between the lattice-induced and the electronic contributions to the linear electro-optic effect [5] and the relative Raman scattering intensities from LO and TO phonons [13]. Experimentally, $C_{1}$ can be derived from measurements of the electro-optic coefficient, by frequency mixing or by Raman scattering.

When all frequencies involved in a nonlinear interaction described by $\chi^{(2)}$ are below or in the vicinity of the phonon resonance, the theoretical description has to be extended by considering higher-order moments of the polarization and the lattice potential. Flytzanis calculated the dispersion of the second-order susceptibility below the phonon resonance on the basis of a microscopic model for the polarizability [14]. He showed that the following 
general expression can be derived [15]:

$$
\begin{aligned}
\chi^{(2)}\left(\omega_{3}, \omega_{2}, \omega_{1}\right)=\chi_{E}^{(2)}[ & 1+C_{1}\left(\frac{1}{D\left(\omega_{1}\right)}+\frac{1}{D\left(\omega_{2}\right)}+\frac{1}{D\left(\omega_{3}\right)}\right)+C_{2}\left(\frac{1}{D\left(\omega_{1}\right) D\left(\omega_{2}\right)}+\frac{1}{D\left(\omega_{1}\right) D\left(\omega_{3}\right)}+\frac{1}{D\left(\omega_{2}\right) D\left(\omega_{3}\right)}\right) \\
& \left.+C_{3} \frac{1}{D\left(\omega_{1}\right) D\left(\omega_{2}\right) D\left(\omega_{3}\right)}\right]
\end{aligned}
$$

The additional terms as compared to Eq. (1) have the following physical background: $C_{2}$ represents the phonon interaction through the second-order lattice dipole moment and $C_{3}$ the contribution from the third-order lattice potential anharmonicity. Besides the relevance for the $\mathrm{THz}$ nonlinear susceptibility these terms are also important for two-phonon sidebands in the infrared absorption, for the phonon decay [14,16], and for a quantitative description of Raman spectra [17]. For a determination of the coefficients $C_{2}$ and $C_{3}$ all frequencies involved have to be below or close above the phonon resonance. However, they cannot be determined independently. The value $3 C_{2}+C_{3}$ has been obtained experimentally as 0.39 from microwave-mixing experiments at $57 \mathrm{GHz}$ for GaAs [4]. SHG below the phonon resonance with an intense and tunable laser source would be a most attractive method for the determination of these coefficients.

In order to elucidate the benefits of this method we plot in Fig. 1 the dispersion of the three contributing terms according to Eq. (2) and their sum [plus 1 according to Eq. (2)] for the case of SHG $\left(\omega_{1}=\omega_{2}, \omega_{3}=2 \omega_{1}\right)$ in GaAs. The parameters used for the calculation are $\omega_{\mathrm{TO}}=$ $8.05 \mathrm{THz}, \quad \gamma=0.29 \mathrm{THz}, \quad \chi_{E}^{(2)}=134 \times 10^{-12} \mathrm{~m} / \mathrm{V}$, $C_{1}=-0.59$ (experimental from Ref. [5]), $C_{2}=0.14$, and $C_{3}=-0.07$ (theoretical from [14], giving a value of $3 C_{2}+C_{3}=0.35$, which is smaller than the experimental value 0.39 from Ref. [4]). A resonance is observed in all three terms of the right-hand side of Eq. (2) at half

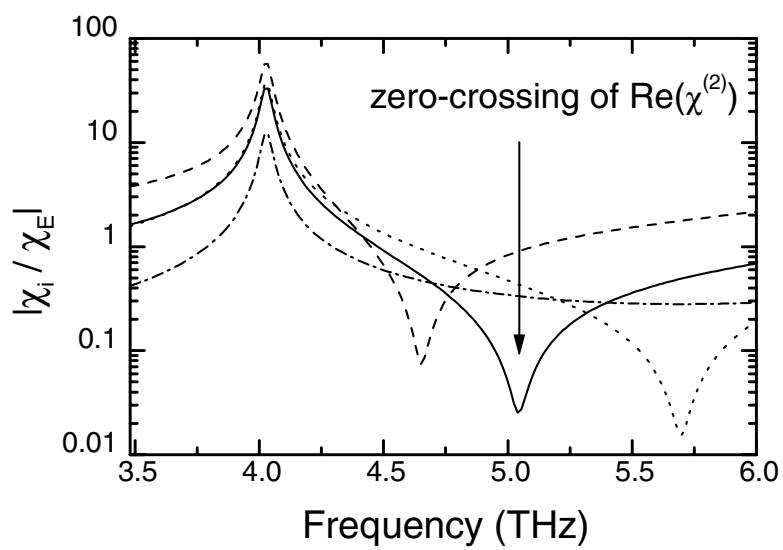

FIG. 1. Calculated contributions to $\chi_{i}^{(2)}$, relative to the electronic second order susceptibility $\chi_{E}^{(2)}$ according to Eq. (2) (absolute values on a logarithmic scale): $C_{1}$ term (dashed), $C_{2}$ term (dotted), $C_{3}$ term (dash-dotted), and the sum of all plus 1 (solid). The arrow indicates the zero crossing in the real part of $\chi^{(2)}$. the TO phonon frequency at $4 \mathrm{THz}$. The maximum value of $\chi^{(2)}$ in resonance exceeds the pure electronic part $\chi_{E}^{(2)}$ by a factor of 33 and has not yet been observed for any III$\mathrm{V}$ compound. Above this resonance a sign reversal occurs in $\chi^{(2)}$. This sign reversal at $5.1 \mathrm{THz}$ should lead to a minimum in the SH power. As proposed earlier [6] an accurate determination of this zero-crossing frequency would thus enable one to determine $C_{2}$ and $C_{3}$ separately [18], and thereby the relative importance of the secondorder dipole moment vs the third-order lattice potential. This method would not require an absolute calibration of the SH conversion efficiency which is a troublesome task in the FIR.

The first frequency doubling experiments below the phonon frequency were performed by Mayer and Keilmann in GaAs using a FIR gas laser operating in the frequency range from 0.6 to $1.7 \mathrm{THz}$ [6]. However, these frequencies were too far away from the predicted resonance $(4 \mathrm{THz})$ to see any resonance enhancement [19]. No values $3 C_{2}$ and $C_{3}$ could be determined due to the uncertainty in the measured $\chi^{(2)}$. The zero crossing of the second-order susceptibility expected around $5.1 \mathrm{THz}$ could also not be observed.

We perform SHG experiments on thin (211) oriented GaAs crystals with thicknesses of 7 to $18 \mu \mathrm{m}$. The effective nonlinear suceptibility for this orientation is $\chi_{\text {eff }}^{(2)}=(2 / \sqrt{3}) \chi_{14}^{(2)}$. The crystals are of homogeneous thickness which can be determined by FIR transmission measurements to within $\pm 2 \mu \mathrm{m}$. Such thin crystals are required by the short coherence length of 10-20 $\mu \mathrm{m}$ associated with the large phase mismatch for SHG in the vicinity of the phonon resonance. The FEL FELIX (Rijnhuizen, The Netherlands) delivers $\mathrm{THz}$ pulses at a macrobunch repetition rate of $10 \mathrm{~Hz}$ and a microbunch repetition rate of $25 \mathrm{MHz}$ with 100 micropulses per macropulse. The radiation frequency is tuned between 4 and $6 \mathrm{THz}$ with a spectral width (FWHM) of 0.2 to $0.25 \mathrm{THz}$ and a micropulse energy between 4 and $8 \mu \mathrm{J}$. The average power of 40 to $80 \mathrm{~mW}$ is low enough to avoid sample heating which could shift the phonon resonance. The radiation was focused by a spherical mirror to a spot with 500 to $650 \mu \mathrm{m}$ diameter (depending on $\lambda$ ) on the GaAs crystal mounted on a metallic aperture of $5 \mathrm{~mm}$ diameter. The SH power was measured with a highsensitivity liquid He cooled Ge:Ga detector and integrated over a macropulse. Two important tasks have to be accomplished to unambiguously measure the SHG from the sample: (i) The FEL beam has to be purified 
from higher harmonics. This is achieved with a $2.7 \mathrm{~mm}$ thick crystalline quartz plate which has an absorption coefficient of $\geq 55 \mathrm{~cm}^{-1}$ in the frequency range from 8.9 to $40 \mathrm{THz}$, thus suppressing higher harmonics down to $\leq 3.5 \times 10^{-7}$, while the transmittance at the fundamental frequency from 4 to $6 \mathrm{THz}$ is approximately $50 \%$. (ii) The fundamental beam transmitted through the sample has to be efficiently blocked before the detector. This is achieved with a double pass through a $2.7 \mathrm{~cm}$ thick $\mathrm{CsBr}$ crystal which has an absorption coefficient of $\geq 7.9 \mathrm{~cm}^{-1}$ in the frequency range from 4 to $5.4 \mathrm{THz}$. The residual transmittance is $3 \times 10^{-19}$, while the $\mathrm{SH}$ power is only weakly attenuated by losses from four reflecting surfaces (each 5.5\%, $n=1.62$ ) and the internal absorption coefficient of $0.1 \mathrm{~cm}^{-1}$ for $\geq 8 \mathrm{THz}$, altogether $66 \%$. The whole setup is placed in a vacuum of $10^{-4}$ mbar in order to avoid water absorption.

Figure 2(a) depicts the power on the detector for a sample of $18 \mu \mathrm{m}$ thickness vs the fundamental frequency. Two FEL power settings, $P_{0}$ and $P_{0} / 2$ are used, achieved by a calibrated broadband attenuator (LASNIX). A separate measurement (not shown) without a sample in the beam path reveals a strongly increasing detector power
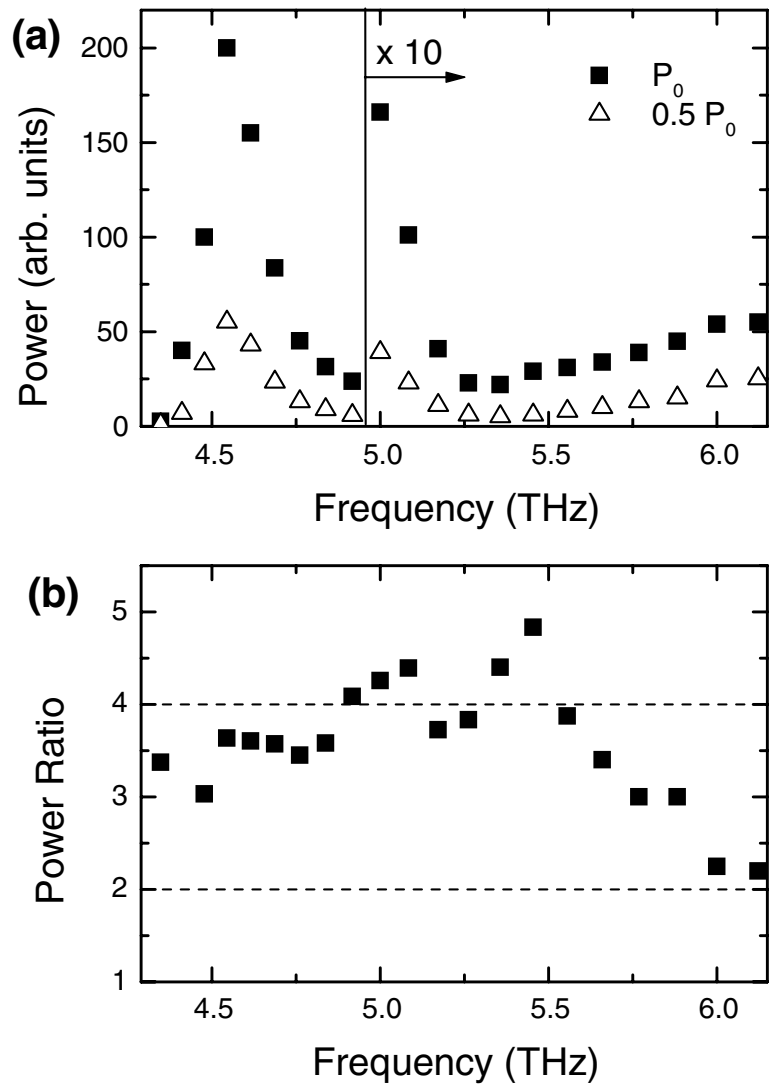

FIG. 2. (a) Detector signal vs fundamental FEL frequency measured with a $18 \mu \mathrm{m}$ thick, (211) oriented GaAs crystal. For frequencies above $4.9 \mathrm{THz}$ the data are multiplied by 10 . (b) Ratio of the detector signal for power settings $P_{0}$ and $P_{0} / 2$ vs fundamental FEL frequency. for frequencies $\geq 5.7 \mathrm{THz}$, where $\mathrm{CsBr}$ starts transmitting the fundamental. In this measurement, a slight increase is also observed below $4.4 \mathrm{THz}$ due to a reduced attenuation of the SH content in the FEL beam by the quartz plate. Fortunately, this signal is strongly attenuated once the GaAs sample is present, because frequencies below $8.8 \mathrm{THz}$ are in the phonon or Reststrahlen band of GaAs (see, e.g., inset of Fig. 3). We can conclude that the setup is sensitive only to SHG between 4.4 and $5.6 \mathrm{THz}$.

Before we discuss the frequency dependence of the detected power in Fig. 2(a) we check its SHG origin. Figure 2(b) shows the ratio of the detector signals for the two power settings, $P_{0}$ and $P_{0} / 2$. Below a frequency of $5.6 \mathrm{THz}$ the ratio is close to 4 as expected for SHG. For frequencies larger than $5.8 \mathrm{THz}$ the ratio drops to 2 which indicates that the fundamental is leaking through the CsBr filter, exceeding the SHG signal. The scattering of the data is due to fluctuations and drift of FEL power [each sweep of Fig. 2(a) takes approximately $20 \mathrm{~min}$ ]. However, changing the fundamental power at a fixed frequency gives ratio values of 4 with errors of $10 \%$ only, for frequencies $<5.6 \mathrm{THz}$. As corroborated from measurements on different sample thicknesses the data shown in Fig. 2(a) are certainly dominated by SHG below $5.6 \mathrm{THz}$.

The SHG power exhibits a maximum at $4.5 \mathrm{THz}$ and drops to a minimum at approximately $5.3 \mathrm{THz}$ [Fig. 2(a)]. Both features are reproduced in samples of different thicknesses (not shown). The maximum at $4.5 \mathrm{THz}$ is the first observation of a resonant enhancement of SHG close to half the phonon energy. The maximum is shifted to somewhat higher frequencies due to the strong absorption of the SHG at $\omega=\omega_{\mathrm{TO}}$ (see calculations below). The minimum observed is a clear indication of a strong drop of $\chi^{(2)}$. Since the expected rise at higher frequencies cannot be certified from the data unambiguously due to insufficient filtering of fundamental FEL power on the detector, this minimum position represents a low-frequency limit only of the zero crossing of $\chi^{(2)}$. Therefore, the zero-crossing frequency is significantly higher than $5.1 \mathrm{THz}$ calculated with the $C_{i}$ 's values used in Fig. 1.

For a comparison we calculate the SH power using Eq. (2) including phase matching, absorption at the fundamental and the SH frequencies, the finite sample thickness, and Fresnel losses [6,7,20]. The dielectric function is calculated from an oscillator model with damping and TO frequency given above and an LO frequency of 8.85 THz. Figure 3 shows the calculated SHG power for three different sets of $C_{i}$ values for a sample thickness of $18 \mu \mathrm{m}$. In addition to the dispersion of $\chi^{(2)}$ shown in Fig. 1 the SHG power is modified by the strong dispersion of the phase-matching condition and the strong absorption of the SH wave close to the TO phonon. The maximum position observed around $4.5 \mathrm{THz}$ is due to a 


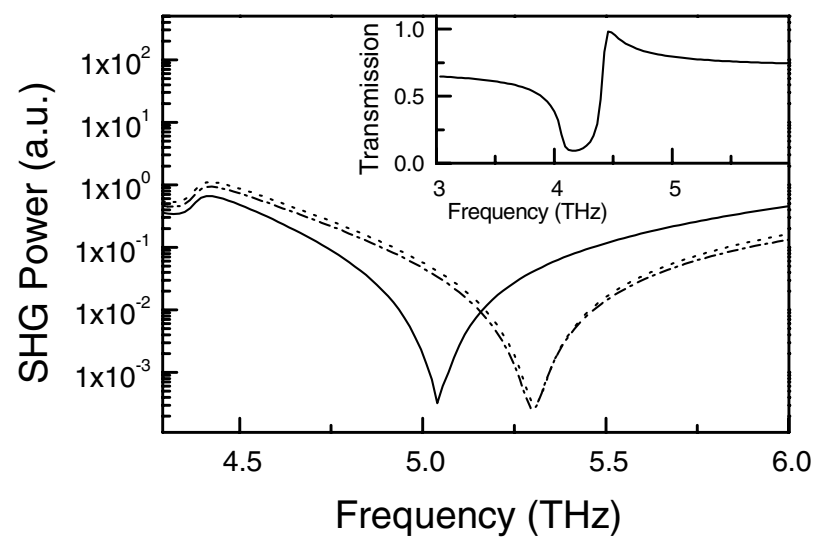

FIG. 3. Calculated SHG power for a $18 \mu \mathrm{m}$ thick GaAs film for 3 different sets of $C_{i}$ 's: $C_{2} / C_{3}=-2.0,3 C_{2}+C_{3}=0.35$ (solid); $C_{2} / C_{3}=-1.23,3 C_{2}+C_{3}=0.35$ (dotted); $C_{2} / C_{3}=$ $-1.3,3 C_{2}+C_{3}=0.39$ (dash-dotted). The inset depicts the transmission coefficient at the $\mathrm{SH}$ frequency vs the fundamental frequency.

maximum transmission of the GaAs crystal at the second harmonic frequency (see inset of Fig. 3). The signal decrease below $4.5 \mathrm{THz}$, likewise, is due to the Reststrahlen minimum transmission. Inserting the value for $\chi^{(2)}(\omega)$ of Fig. 1 (with $C_{1}=-0.59, C_{2}=0.14, C_{3}=-0.07$ ) predicts the SHG minimum and a zero crossing at $5.1 \mathrm{THz}$. Using the experimental value $3 C_{2}+C_{3}=0.39$ (while keeping the ratio $C_{2} / C_{3}$ fixed at the theoretical value of -2.0 , i.e., $C_{2}=0.156, C_{3}=-0.078$ ) would result in a slightly smaller zero-crossing frequency of $5.08 \mathrm{THz}$. In order to match the zero crossing to the experimentally observed value of $5.3 \mathrm{THz}$, the ratio of $C_{2} / C_{3}$ has to be changed significantly from -2.0 to values between -1.23 and -1.30 with corresponding values of $3 C_{2}+C_{3}$ between 0.35 and 039 , respectively. We thus conclude that the contribution of the phonon interaction through the third-order lattice potential anharmonicity $\left(C_{3}\right)$ is significantly larger than calculated by theory [14], relative to the contribution from the secondorder lattice dipole moment $\left(C_{2}\right)$.

In summary, we observed the resonance of the secondorder nonlinear susceptibility of GaAs at half the phonon frequency. A vanishing SHG power between half the phonon frequency and the phonon frequency has been observed as predicted. This minimum in the SHG is based on the cancellation of the electronic and ionic parts - including higher-order polarizations and lattice potential anharmonicity - of the susceptibility. The determination of this zero crossing allows us to derive new weights of relative contributions to the second-order nonlinear susceptibility. We believe that the presented method is valuable for the determination of these coefficients in other technologically important materials.

We thank J. Kuhl and A. F. G. van der Meer for fruitful discussions. We gratefully acknowledge the support by the Stichting voor Fundamenteel Onderzoek der Materie (FOM) in providing the required beam time on FELIX and highly appreciate the skillful assistance by the FELIX staff. This work was supported in part under the "Access to research infrastructure" action of the European Community.

[1] P. A. Franken, A. E. Hill, C.W. Oeters, and G. Weinreich, Phys. Rev. Lett. 7, 118 (1961).

[2] F. Keilmann, Infrared Phys. 31, 373 (1991).

[3] W. L. Faust and C. H. Henry, Phys. Rev. Lett. 17, 1265 (1966); W. L. Faust, C. H. Henry, and R. H. Eick, Phys. Rev. 173, 781 (1968).

[4] G. D. Boyd, T. J. Bridges, M. A. Pollack, and E. H. Turner, Phys. Rev. Lett. 26, 387 (1971).

[5] W. D. Johnston and I. P. Kaminov, Phys. Rev. 188, 1209 (1969).

[6] A. Mayer and F. Keilmann, Phys. Rev. B 33, 6954 (1986).

[7] M. Barmentlo, G.W. 't Hooft, E. R. Eliel, E.W. M. van der Ham, Q. H. F. Vrehen, A. F. G. van der Meer, and P.W. van Amersfoort, Phys. Rev. A 50, R14 (1994).

[8] R. Köhler, A. Tredicucci, F. Beltram, H. E. Beere, E. H. Linfield, A. G. Davies, D. A. Ritchie, R. C. Iotti, and F. Rossi, Nature (London) 417, 156 (2002).

[9] J. J. Wynne and N. Bloembergen, Phys. Rev. 188, 1211 (1969).

[10] J. H. McFee, G. D. Boyd, and P. H. Schmidt, Appl. Phys. Lett. 17, 57 (1970).

[11] M. M. Choy and R. L. Byer, Phys. Rev. B 14, 1693 (1976).

[12] C. C. Shih and A. Yariv, J. Phys. C 15, 825 (1982).

[13] A. Pinczuk and E. Burstein, in Light Scattering in Solids I, edited by M. Cardona, Topics in Applied Physics Vol. 8 (Springer, Berlin, 1983), 2nd ed., pp. 23-78.

[14] C. Flytzanis, Phys. Rev. B 6, 1264 (1972).

[15] We restrict ourselves to a scalar notation since the $\chi^{(2)}$ tensor of GaAs has only one nonvanishing element $\chi_{14}^{(2)}$.

[16] C. Flytzanis, Phys. Rev. Lett. 29, 772 (1972).

[17] S. Go, H. Bilz, and M. Cardona, Phys. Rev. Lett. 34, 580 (1975).

[18] The experimental determination of $3 C_{2}+C_{3}$ only does not allow a determination of the ratio $C_{2} / C_{3}$, for which only the theoretical value of -2.0 is known. However, from the experimentally observed zero crossing we can derive a unique range of $C_{2}$ and $C_{3}$ values.

[19] The onset of a resonance enhancement of SHG was, however, observed at $2 \mathrm{THz}$ in $\mathrm{LiTaO}_{3}$ which has a lower phonon frequency, $\omega_{\mathrm{TO}}=4.3 \mathrm{THz}[6]$.

[20] N. Bloembergen and P. S. Pershan, Phys. Rev. 128, 606 (1962). 